\title{
Dynamical gluon mass at nonzero temperature in instanton vacuum model
}

\author{
M. Musakhanov, Sh. Baratov, and N. Rakhimov \\ Theoretical Physics Dept, Uzbekistan National University, Tashkent 100174, Uzbekistan
}

(Received 11 February 2019; published 8 April 2019)

\begin{abstract}
In the framework of the instanton liquid model (ILM), we consider thermal modifications of gluon properties in different scenarios of temperature $(T)$ dependence of the average instanton size $\bar{\rho}(T)$ and instanton density $n(T)$ known from the literature. Due to interactions with instantons, the gluons acquire the dynamical temperature-dependent "electric" gluon mass $M_{\mathrm{el}}(q, T)$. We find that at small momenta and zero temperature $M_{\mathrm{el}}(0,0) \approx 362 \mathrm{MeV}$ for the phenomenological values of $\bar{\rho}(0)=1 / 3 \mathrm{fm}$ and $n(0)=1 \mathrm{fm}^{-4}$. However, the $T$ dependence of the mass is very sensitive to the temperature dependence of the instanton vacuum parameters $\bar{\rho}(T)$ and $n(T)$ : it is very mild in the case of the lattice-motivated dependence and decreases steeply in the whole range with theoretical parametrization. We find that the region $0<T<T_{c}$ ILM is able to reproduce lattice results for the dynamical gluon mass.
\end{abstract}

DOI: 10.1103/PhysRevD.99.074005

\section{INTRODUCTION}

Gluodynamics at nonzero temperature $T(\equiv 1 / \beta)$ is described by the partition function

$Z=\int D A_{\mu} \exp \left\{-\frac{1}{2 g^{2}} \int_{0}^{\beta} d x_{4} \int d^{3} x \operatorname{tr} F_{\mu \nu} F_{\mu \nu}\right\}$

where $F_{\mu \nu}=\partial_{\mu} A_{\nu}-\partial_{\nu} A_{\mu}-i\left[A_{\mu}, A_{\nu}\right]$, and the gauge field $A_{\mu}$ satisfies the periodic condition $A_{\mu}\left(\vec{x}, x_{4}+\beta\right)=A_{\mu}\left(\vec{x}, x_{4}\right)$. The extension of the zero-temperature instanton solution [1] — the caloron (found in Ref. [2])—-has the form

$$
\begin{gathered}
A_{\mu}^{I}=\Pi \bar{\eta}_{\mu \nu}^{a}\left(\tau_{a} / 2 i\right) \partial_{\nu} \Pi^{-1}, \\
F_{\mu \nu}=\frac{1}{2} \Pi(\tau \partial) \bar{\eta}_{\mu \nu}^{a}\left(\tau_{a} / 2 i\right)\left(\tau^{+} \partial\right) \Pi^{-1}, \quad \Pi^{-1} \partial^{2} \Pi=0, \\
\Pi(r, t)=1+\frac{\pi \rho^{2}}{\beta r} \sinh \frac{2 \pi r}{\beta} /\left(\cosh \frac{2 \pi r}{\beta}-\cos \frac{2 \pi t}{\beta}\right) \\
=1+\sum_{n=-\infty}^{\infty} \frac{\rho^{2}}{r^{2}+(t-n \beta)^{2}},
\end{gathered}
$$

where $r=|\vec{x}|, t=x_{4}$, and $\tau_{\mu}=(\vec{\tau}, i)$. At small distances $r, t \ll \beta$ the profile $\Pi(x)$ may be approximated as

*musakhanov@gmail.com

Published by the American Physical Society under the terms of the Creative Commons Attribution 4.0 International license. Further distribution of this work must maintain attribution to the author(s) and the published article's title, journal citation, and DOI. Funded by SCOAP ${ }^{3}$.

$$
\Pi(x) \approx \Pi_{0}(x)=\left(1+\frac{\lambda^{2}}{3}\right)+\rho^{2} / x^{2},
$$

where $\lambda=\pi \rho / \beta$, so the gluon field has an instanton-like shape with modified instanton size,

$$
A_{\mu}^{I, a}=\frac{2 \rho^{\prime 2}}{x^{2}} \frac{\bar{\eta}_{\mu \nu}^{a} x_{\nu}}{\left(x^{2}+\rho^{\prime 2}\right)}, \quad \rho^{\prime 2}=\rho^{2} /\left(1+\frac{\lambda^{2}}{3}\right) .
$$

In fact, the accuracy of the approximation (4) is about $1 \%$ up to $r, t \sim \beta$. The extension of the instanton vacuum liquid model (ILM) [3-5] to nonzero temperature in this regime is straightforward and might be encoded in the temperature dependences of the main parameters of the model: the average instanton size $\bar{\rho}(T)$ and average instanton density $n(T)=N T / V_{3}=1 / R^{4}(T)$, where $N$ is the total number of instantons [6]. Both $\bar{\rho}(T)$ and $n(T)$ in the ILM are homogeneously decreasing functions of $T$.

But the simplified approximation (4) does not describe a nontrivial phase transition near the critical temperature $T_{\mathrm{c}} \sim \Lambda_{\mathrm{QCD}}$. Indeed, for $T<T_{\mathrm{c}}$ all color objects are bound into colorless hadrons. The heat bath predominantly consists of weakly interacting pions, so the $T$ dependence of the instanton density $n(T)$ should be rather mild, which agrees with the expectation of almost constant $T$ dependence, $n(T)=n_{0}\left(1+O\left(T^{2} /\left(6 f_{\pi}^{2}\right)\right)\right)$ [5]. However, this behavior changes during phase transitions, and the expected instanton density $n(T)$ should be exponentially suppressed at large temperatures, $T \gtrsim T_{\mathrm{c}}$ [5].

The extension of the ILM that is able to describe the phase transition from a confined to a deconfined phase near the critical temperature $T_{\mathrm{c}}$ is the so-called dyon-instanton liquid model [7]. The authors of Ref. [7] concluded later in 
Ref. [8] that at very low temperature, the semiclassical description of the Yang-Mills state reconciles the instanton liquid model without confinement with the 't HooftMandelstam proposal of confinement. In the former, the low-temperature thermal state is composed of a liquid of instantons and anti-instantons, while in the latter it is a superfluid of monopoles and antimonopoles.

The temperature dependence of the QCD vacuum model might be tested by comparison with results of lattice simulations. For example, for the "electric" gluon mass $M_{\mathrm{el}}(T)$ in the framework of lattice QCD [9-12], it was found that for $T \geq T_{\mathrm{c}}$ the linear dependence $M_{\mathrm{el}}(T) \sim T$ is consistent with Debye screening and has a minimum at $T \sim T_{\mathrm{c}}$, whereas for $T \leq T_{\mathrm{c}}$ the electric mass $M_{\mathrm{el}}(T)$ is a rather slowly decreasing function of temperature $T$. This behavior might be naturally explained in the framework of the ILM, which predicts the temperature dependence as

$$
M_{\mathrm{el}} \sim(\operatorname{packing} \operatorname{parameter}(T))^{1 / 2} \bar{\rho}^{-1}(T)=\bar{\rho}(T) n^{1 / 2}(T),
$$

i.e., a decreasing function of temperature at $T \leq T_{\mathrm{c}}$. Combined with the perturbative one-loop thermal gluon contribution to the gluon propagator, which increases with temperature as $M_{\text {pert,el }}(T) \sim T$, this model is able to reproduce lattice results for the dynamical gluon mass $[9,12]$.

There are two major technical challenges in the calculation of the gluon propagator in the ILM framework: the zero-mode problem (fluctuations along the instanton collective coordinates), and averaging over the collective coordinates of all instantons. We address the former using the approach of Ref. [14], while for the latter we extend Pobylitsa's approach [15], applied earlier by us for the gluons at $T=0$ [16], and consider in this paper its further extension for the ILM averaged gluon propagator at $T \neq 0$.

The paper is structured as follows. In Sec. II we review briefly the formulation of the ILM at nonzero temperature $T \neq 0$ and discuss the temperature dependence of the main instanton vacuum parameters. In Sec. III we consider a simplified case and evaluate the propagator of the scalar color-octet particle (which we call the "scalar gluon") in the instanton background at nonzero temperature. This allows us to get several important results which will be used later. In Sec. IV we consider the case of a real gluon and evaluate the propagator at nonzero temperature. We extract the electric mass $M_{\mathrm{el}}$ and compare it with lattice results. Finally, in Sec. V we draw our conclusions.

\section{VARIATIONAL ESTIMATES IN THE ILM AT $T \neq 0$}

The application of Feynman's variational principle to the QCD vacuum filled with an instanton gas leads to the ILM [3], which was generalized to nonzero temperatures in Ref. [6]. The main variational ingredients of this approach are the instanton size distribution function $\mu(\rho, T, n)$ and the instanton density $n(T)[6,6,17]$. The ILM instanton size distribution function is closely related to the thermal single instanton one-loop distribution function [18],

$$
\begin{gathered}
d(\rho, T)=C \rho^{b-5} \exp \left(-A_{N_{\mathrm{c}}} T^{2}\right), \quad C=\mathrm{const}, \\
\mu(\rho, T, n)=C \rho^{b-5} \exp \left[-\Phi(n, T) \rho^{2}\right], \\
\Phi(n, T)=\frac{1}{2} A_{N_{\mathrm{c}}} T^{2}+\left[\frac{1}{4} A_{N_{\mathrm{c}}}^{2} T^{4}+\nu \bar{\beta} \gamma^{2} n\right]^{1 / 2},
\end{gathered}
$$

where

$$
\begin{aligned}
A_{N_{\mathrm{c}}} & =\frac{1}{3}\left[\frac{11}{6} N_{\mathrm{c}}-1\right] \pi^{2}, \quad b=\frac{11}{3} N_{\mathrm{c}}, \nu=\frac{b-4}{2}, \\
\bar{\beta} & =-b \log (\Lambda \bar{\rho}), \quad \gamma^{2}=\frac{27 \pi^{2} N_{\mathrm{c}}}{4\left(N_{\mathrm{c}}^{2}-1\right)}
\end{aligned}
$$

and

$$
\begin{aligned}
& \bar{\rho}^{2}(T, n)=\frac{1}{\mu_{0}(T, n)} \int_{0}^{\infty} d \rho \mu(\rho, T, n) \rho^{2}, \\
& \mu_{0}(T, n)=\int_{0}^{\infty} d \rho \mu(\rho, T, n) .
\end{aligned}
$$

The result (8) might be obtained by maximizing the variational ILM partition function [6]

$$
\begin{aligned}
Z & \geq Z_{1}(\mu, n) \exp \left(-\left\langle E-E_{1}\right\rangle\right), \\
Z_{1}(\mu, n) & =\frac{1}{(N / 2 !)^{2}}\left(\frac{2 \mu_{0}(T, n) V}{N}\right)^{N}, \\
\left\langle E-E_{1}\right\rangle & =-\frac{N}{2} \bar{\beta} \gamma^{2} n \rho^{2} 2
\end{aligned}
$$

with respect to the parameter $\mu$. The minimization of the free energy $F=-T / V_{3} \log Z$ by variation over $n$ leads to the equation for the density,

$$
n(T)=2 \mu_{0}(n, T) .
$$

The variational estimates demonstrate that $\bar{\rho}(T)$ and $n(T)$ are decreasing functions of temperature $T$ (see Fig. 1) due to the exponential factor in $d(\rho, T) \sim A_{N_{\mathrm{c}}}$. On the other hand, lattice data show that the instanton density $n$ is not modified by temperature up to the critical temperature $T_{\mathrm{c}}$ [19]. In numerical simulations of the ILM [5] it was suggested to interpolate between no suppression $\left[A_{N_{\mathrm{c}}}=0\right.$ in Eq. (9)] below $T_{\mathrm{c}}$ and full suppression $\left[A_{N_{\mathrm{c}}} \neq 0\right.$ in Eq. (9)] above $T_{\mathrm{c}} \sim 150 \mathrm{MeV}$, with a width $\Delta T=0.3 T_{\mathrm{c}}$ to be in agreement with the lattice results [19]. We follow this suggestion and repeat the calculations with the modification in Eq. (9) as $A_{N_{\mathrm{c}}} \rightarrow A_{N_{\mathrm{c}}} \Theta_{\Delta y}\left(y-y_{\mathrm{c}}\right)$, where $y=\bar{\rho}_{0} T$, 

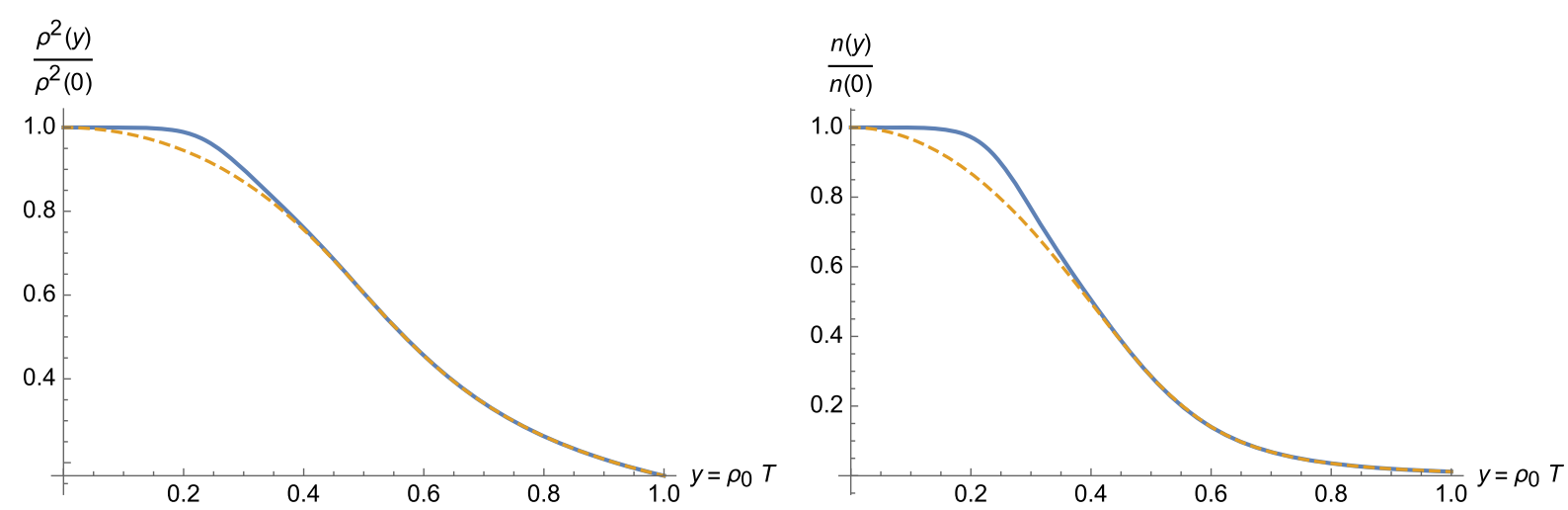

FIG. 1. The (normalized to unity) temperature dependences of the instanton size $\bar{\rho}^{2}(y) / \bar{\rho}^{2}(0)$ (left) and instanton density $n(y) / n(0)$ (right) obtained with variational estimates from Refs. [5,6,17]). In both plots we use the notation $y=\bar{\rho}_{0} T$ and the phenomenological values $\bar{\rho}(0)=1 / 3 \mathrm{fm}$ and $n(0)=1 \mathrm{fm}^{-4}$ for the estimates. The solid line corresponds to values with the modification $A_{N_{\mathrm{c}}} \rightarrow$ $A_{N_{\mathrm{c}}} \Theta_{\Delta y}\left(y-y_{\mathrm{c}}\right)$ in Eq. (9), where $\Theta_{\Delta y}\left(y-y_{\mathrm{c}}\right)$ is the smooth interpolating step-like function (14) with a width $\Delta T=0.3 T_{\mathrm{c}}$ [5]. Dashed lines correspond to values with $A_{N_{\mathrm{c}}}=$ const in Eq. (9) (full suppression) for all $T$.

$y_{\mathrm{c}}=\bar{\rho}_{0} T_{\mathrm{c}}=0.25 \sim T_{\mathrm{c}}=150 \mathrm{MeV}, \Delta y=\bar{\rho}_{0} \Delta T_{\mathrm{c}}=0.075 \sim$ $\Delta T=0.3 T_{\mathrm{c}}$, and we introduce a smooth interpolation with a step-like function of width $\Delta y$,

$$
\Theta_{\Delta y}(z)=1 / 2[1+\tanh (z / \Delta y)]
$$

The temperature dependences of $\overline{\rho^{2}}(y) / \overline{\rho^{2}}(0)$ and $n(y) / n(0)$ are shown in Fig. 1.

\section{COLOR-OCTET SCALAR PROPAGATOR AT NONZERO TEMPERATURE}

We start from the scalar massless field $\phi$ belonging to the adjoint representation, which is the same as a physical gluon. We have to find its propagator in the external classical gluon field of instanton gas $A_{\mu}=\sum_{I} A_{\mu}^{I}\left(\gamma_{I}\right)$, where $A_{\mu}^{I}\left(\gamma_{I}\right)$ is a generic notation for the QCD (anti-) instanton, and $\gamma_{I}$ stands for all of the relevant collective coordinates: the position in Euclidean four-dimensional space $z_{I}$, the size $\rho_{I}$, and the $S U\left(N_{\mathrm{c}}\right)$ color orientation $U_{I}$ $\left(4 N_{\mathrm{c}}\right.$ collective coordinates in total). The averaging over the instanton collective coordinates includes the integral over the instanton position $\int d^{4} z \equiv \int_{V_{3}} d^{3} z_{I} \int_{0}^{\beta} d z_{I, 4}$. In view of the periodicity of the fields $\phi(\vec{x}, t+\beta)=\phi(\vec{x}, t)$ at nonzero temperature, we may restrict the integration over $t$ to the period $\beta$, so the effective action takes the form

$$
S_{\phi}=\int_{V_{3}} d^{3} x \int_{0}^{\beta} d t \phi^{\dagger}(\vec{x}, t) P^{2} \phi(\vec{x}, t)
$$

where $P_{\mu}=p_{\mu}+A_{\mu}$ (in the coordinate representation $p_{\mu}=i \partial_{\mu}$ ). The color-octet scalar propagator in the field of instanton gas is given by

$$
\begin{aligned}
\Delta & =(p+A)^{-2}=\left(p^{2}+\sum_{i}\left(\left\{p, A_{i}\right\}+A_{i}^{2}\right)+\sum_{i \neq j} A_{i} A_{j}\right)^{-1}, \\
\Delta_{0} & =p^{-2} .
\end{aligned}
$$

It is convenient to also introduce the propagator in the field of an individual instanton,

$$
\Delta_{i}=P_{i}^{-2}=\left(p^{2}+\left\{p, A_{i}\right\}+A_{i}^{2}\right)^{-1},
$$

and in the instanton gas background when the overlaps $\sim \sum_{i \neq j} A_{i} A_{j}$ are disregarded,

$$
\tilde{\Delta}=\left(p^{2}+\sum_{i}\left(\left\{p, A_{i}\right\}+A_{i}^{2}\right)\right)^{-1} .
$$

There are no zero modes in $\Delta_{i}^{-1}=P_{i}^{2}$ or $\Delta^{-1}=P^{2}$, which implies the existence of the inverse operators $\Delta_{i}$ and $\Delta$. Our aim is to find the propagator averaged over instanton collective coordinates $\bar{\Delta} \equiv\langle\Delta\rangle=\int D \gamma \Delta$. In coordinate space the propagator $\Delta$ and free propagator $\Delta_{0}$ must be periodic functions of time with period $\beta$. In what follows we will use the notation $\Delta\left(x, x^{\prime}\right) \equiv\left\langle x|\Delta| x^{\prime}\right\rangle$ for the matrix element of the operator $\Delta$ between Fock states labeled by spacetime coordinates $|x\rangle \equiv|\vec{x}, t\rangle=|\vec{x}\rangle|t\rangle$ (the same for $\left.\left|x^{\prime}\right\rangle\right)$ and $\left[x_{\mu}, p_{\nu}\right]=i \delta_{\mu \nu}$ [20]. These states form a complete orthonormalized set, $\sum_{t}|t\rangle\langle t|=1,\left\langle t^{\prime} \mid t\right\rangle=\delta\left(t^{\prime}-t\right)$. Also, we define the step operator $\left\langle t^{\prime}|\Theta| t\right\rangle=\Theta\left(t^{\prime}-t\right)$. In view of $\frac{d}{d t^{\prime}} \Theta\left(t^{\prime}-t\right)=\delta\left(t^{\prime}-t\right)$, we may conclude that $\Theta^{-1} \equiv \frac{d}{d t}$. The time-periodic state with period $\beta$ may be represented in terms of states $|t\rangle$ as

$$
\begin{aligned}
\left|t_{\beta}\right\rangle & \equiv \sum_{n=-\infty}^{\infty}|t-n \beta\rangle \\
\left\langle t^{\prime} \mid t_{\beta}\right\rangle & =\delta\left(t^{\prime}-t_{\beta}\right) \equiv \sum_{n=-\infty}^{\infty} \delta\left(t^{\prime}-(t-n \beta)\right) .
\end{aligned}
$$


Now the evaluation of the propagator $\Delta=\left(P^{2}\right)^{-1}$ is straightforward. Since $\left\langle t^{\prime}\left|P^{2}\right| t_{\beta}\right\rangle=\delta\left(t^{\prime}-t_{\beta}\right)\left(\vec{P}^{2}+\left(i \frac{\partial}{\partial t^{\prime}}+A_{4}\right)^{2}\right)$, we have the equation in the form

$$
\begin{aligned}
\left(\vec{P}^{2}+\left(i \frac{\partial}{\partial t^{\prime}}+A_{4}\right)^{2}\right)\left\langle t^{\prime}|\Delta| t_{\beta}\right\rangle & =\delta\left(t^{\prime}-t_{\beta}\right) \\
\Rightarrow\left\langle\vec{x}^{\prime}, t^{\prime}|\Delta| \vec{x}, t_{\beta}\right\rangle & =\sum_{n=-\infty}^{\infty} \Delta\left(\vec{x}^{\prime}, t^{\prime} \mid \vec{x}, t-n \beta\right),
\end{aligned}
$$

where $\Delta\left(\vec{x}^{\prime}, t^{\prime} \mid \vec{x}, t-n \beta\right)$ is a usual zero-temperature $(T=0)$ aperiodic propagator. For physical applications we need to average the propagator $\Delta$ over the collective coordinates of all instantons, $\bar{\Delta}=\langle\Delta\rangle=\int D \gamma \Delta$. We follow the procedure developed in our previous paper [16], where the approach [15] derived for the quark correlators was extended to the gluon case. We start by averaging over the collective coordinates of the operator $\overline{\tilde{\Delta}}=\langle\tilde{\Delta}\rangle$ [see Eq. (16)]. Since the Pobylitsa equations $[15,16]$ are written in operator form, they can be easily extended to the $T \neq 0$ case just by calculating the matrix elements of the propagator $\tilde{\Delta}$ with periodic states $\left|t_{\beta}\right\rangle$ on the right side.

Since the instanton gas is dilute [namely, the packing parameter $\left.\rho^{4} n \sim(1 / 3)^{4}=1.2 \times 10^{-2} \ll 1\right]$, we may develop a systematic expansion over the parameter $n$. The expansion of the inverse propagator up to first-order $\mathcal{O}(n)$ terms has the form

$$
\begin{aligned}
\overline{\tilde{\Delta}}^{-1}-\Delta_{0}^{-1} & =\left\langle\sum_{i}\left\{\Delta_{0}+\left(\Delta_{i}^{-1}-\Delta_{0}^{-1}\right)^{-1}\right\}^{-1}\right\rangle \\
& =N \Delta_{0}^{-1}\left(\bar{\Delta}_{I}-\Delta_{0}\right) \Delta_{0}^{-1}+\mathcal{O}\left(n^{2}\right),
\end{aligned}
$$

where $\bar{\Delta}_{I}=\int d \gamma_{I} \Delta_{I}$ is the propagator in the field of an individual instanton averaged over its collective degrees of freedom. To the same expansion order, we may approximate the inverse propagator as $\bar{\Delta}^{-1}=\overline{\tilde{\Delta}}^{-1}=p^{2}+M_{s}^{2}$, where we introduced the squared dynamical coloroctet scalar mass operator $M_{s}^{2}$ whose matrix elements are given by

$$
\left\langle t^{\prime}\left|M_{S}^{2}\right| t_{\beta}\right\rangle \delta_{a b}=N p^{2}\left(\left\langle t^{\prime}\left|\bar{\Delta}_{I}^{a b}\right| t_{\beta}\right\rangle-\left\langle t^{\prime}\left|\Delta_{0}^{a b}\right| t_{\beta}\right\rangle\right) p^{2} .
$$

According to Ref. [18], the periodic color-octet scalar propagator in the instanton field (3) is given by

$$
\begin{aligned}
\Delta_{I}^{a b}(x, y)= & \Delta_{0}^{a b}(x, y)+\Delta_{1}^{a b}(x, y)+\Delta_{2}^{a b}(x, y), \\
\Delta_{0}^{a b}(x, y) & =\frac{1}{2} \operatorname{tr}\left(\frac{\tau_{a} F(x, y) \tau_{b} F(y, x)}{\Pi(x) 4 \pi^{2}(x-y)^{2} \Pi(y)}\right), \\
F(x, y)= & 1+\sum_{m} \frac{\rho^{2}\left(\tau x_{m}\right)\left(\tau^{\dagger} y_{m}\right)}{x_{m}^{2} y_{m}^{2}} \\
& \times\left(x_{m} \equiv x-m \beta \hat{t}, y_{m} \equiv y-m \beta \hat{t}\right),
\end{aligned}
$$

$$
\begin{aligned}
& \Delta_{1}^{a b}(x, y)= \frac{1}{2} \operatorname{tr}\left(\sum_{m}, \frac{\tau_{a} F\left(x, y_{m}\right) \tau_{b} F\left(y_{m}, x\right)}{\Pi(x) 4 \pi^{2}\left(x-y_{m}\right)^{2} \Pi(y)}\right), \\
& \Delta_{2}^{a b}(x, y)=\sum_{m} \frac{C^{a b}\left(x, y_{m}\right)}{\Pi(x) 4 \pi^{2} \Pi(y)}, \\
& C^{a b}(x, y)=\sum_{r \neq s} \frac{2 \Phi_{r s}^{a}(x) \Phi_{r s}^{b}(y)}{\beta^{2}(r-s)^{2}} \\
&-\sum_{r \neq s} \sum_{t \neq u} \frac{\rho^{2} \Phi_{r s}^{a}(x)}{\beta^{2}(r-s)^{2}} \frac{\Phi_{t u}^{b}(y)}{\beta^{2}(t-u)^{2}} h_{r s, t u}, \\
& \Phi_{r s}^{a}(x)=\frac{\rho^{2} \beta(r-s) x^{a}}{x_{r}^{2} x_{s}^{2}}, \\
& \sum_{m} C^{a b}\left(x, y_{m}\right)=\sum_{m} \sum_{r \neq s} \frac{2 \Phi_{r s}^{a}(x) \Phi_{r s}^{b}\left(y_{m}\right)}{\beta^{2}(r-s)^{2}} \\
&=\sum_{r \neq s} \frac{\rho^{2} x^{a}}{x_{r}^{2} x_{s}^{2}} \sum_{m} \frac{\rho^{2} y^{b}}{y_{r+m}^{2} y_{s+m}^{2}} .
\end{aligned}
$$

Combining Eqs. (28) and (29), we can simplify Eq. (27) as

$\Delta_{2}^{a b}(x, y)=\sum_{r \neq s} \frac{\rho^{2} x^{a}}{x_{r}^{2} x_{s}^{2}} \sum_{m} \frac{\rho^{2} y^{b}}{y_{r+m}^{2} y_{s+m}^{2}} \frac{1}{\Pi(x) 4 \pi^{2} \Pi(y)}$.

At short distances $r \sim t \leq \beta$ the caloron field becomes instanton-like [Eq. (5)] with a modified instanton radius $\rho^{\prime 2}=\rho^{2} /\left(1+1 / 3 \lambda^{2}\right)$, and $\lambda=\pi \rho / \beta$. In this region we can simplify the first term in Eq. (23) as

$\Delta_{I, 0}^{a b}=\frac{1}{2} \operatorname{tr}\left(\frac{\tau_{a} F_{0}(x, y) \tau_{b} F_{0}(y, x)}{4 \pi^{2}(x-y)^{2} \Pi_{0}(x) \Pi_{0}(y)}\right), \quad \Pi_{0}(x)=\frac{x^{2}+\rho^{\prime 2}}{x^{2}}$,

$\tau_{\mu} \equiv(\vec{\tau}, i), \quad \tau_{\mu}^{\dagger}=(\vec{\tau},-i), \quad \tau_{\mu} \tau_{\nu}^{+}=\delta_{\mu \nu}+i \bar{\eta}_{a \mu \nu} \tau_{a}$,

$$
\begin{aligned}
F_{0}(x, y) & =1+\rho^{\prime 2} \frac{(\tau x)\left(\tau^{+} y\right)}{x^{2} y^{2}} \\
& =1+\rho^{\prime 2} \frac{(x y)}{x^{2} y^{2}}+\rho^{\prime 2} \frac{i \bar{\eta}_{a \mu \nu} \tau_{a} x_{\mu} y_{\nu}}{x^{2} y^{2}},
\end{aligned}
$$

where $\bar{\eta}_{a \mu \nu}=-\bar{\eta}_{a \nu \mu}$ is the 't Hooft symbol. As will be shown below, the contribution of the terms $\Delta_{1}^{a b}(x, y)$ and $\Delta_{2}^{a b}(x, y)$ in Eq. (23) is small and can be neglected.

The collective degrees of freedom (the center of the instanton position $z$ and orientation $U$ ) in Eq. (31) can be introduced by shifting the arguments $x \rightarrow x-z$ and $y \rightarrow y-z$ and color rotation factors $\Delta_{I}^{a b} \rightarrow$ $O^{a b} O^{a^{\prime} b^{\prime}} \Delta_{I}^{b b^{\prime}}$, where $O^{a b}$ are the color rotation matrices in the adjoint representation. $\left[O^{a b}\right.$ are related to the color rotation matrices the in fundamental representation by 
$O^{a b}=\operatorname{tr}\left(U^{\dagger} t^{a} U t^{b}\right)$, where $t_{a}$ are $S U\left(N_{\mathrm{c}}\right)$ matrices.] The averaging over the collective coordinates reduces to integration over the instanton center $\left(\int_{0}^{\beta} d z_{4} \int_{V_{3}} d^{3} z\right)$ and color orientation $\left(\int d O\right)$. The latter integral can be evaluated analytically using the well-known identities [5] $\int d O O^{a b} O^{a b^{\prime}}=\delta_{b b^{\prime}}, \int d O O^{a b} O^{a^{\prime} b^{\prime}}=\left(N_{\mathrm{c}}^{2}-1\right)^{-1} \delta_{a a^{\prime}} \delta_{b b^{\prime}}$ and $\int d O O^{a b} \bar{\eta}_{b \mu \nu} O^{a^{\prime} b^{\prime}} \bar{\eta}_{b^{\prime} \mu^{\prime} \nu^{\prime}}=\left(N_{\mathrm{c}}^{2}-1\right)^{-1} \delta_{a a^{\prime}}\left(\delta_{\mu \mu^{\prime}} \delta_{\nu \nu^{\prime}}-\delta_{\mu \nu^{\prime}} \delta_{\nu \mu^{\prime}}\right)$.
The contribution of $\Delta_{I, 0}^{a b}$ to the color-octet scalar dynamical mass operator (22) is given by

$$
M_{s, 0}^{2} \delta_{a b}=N p^{2}\left(\bar{\Delta}_{I, 0}^{a b}-\Delta_{0}^{a b}\right) p^{2}
$$

so for the expression in parentheses in Eq. (34) we obtain, after collective coordinate averaging in the coordinate representation,

$$
\begin{aligned}
\bar{\Delta}_{I, 0}^{a a^{\prime}}(x, y)-\Delta_{0}^{a a^{\prime}}(x, y) & =\int d^{4} z d O O^{a c} O^{a^{\prime} c^{\prime}}\left(\Delta_{I, 0}^{c c^{\prime}}\left(x^{\prime}, y^{\prime}\right)-\Delta_{0}^{c c^{\prime}}\left(x^{\prime}, y^{\prime}\right)\right)\left(x^{\prime} \equiv x-z, y^{\prime} \equiv y-z\right), \\
& =\delta_{a a^{\prime}} \int d^{4} z\left[\frac{3 \rho^{\prime 2}}{4 \pi^{2}\left(N_{\mathrm{c}}^{2}-1\right)} f_{1}\left(x^{\prime}\right) f_{1}\left(y^{\prime}\right)+\frac{2 \rho^{\prime 4}}{N_{\mathrm{c}}^{2}-1} f_{2}\left(x^{\prime}\right) g\left(x^{\prime}-y^{\prime}\right) f_{2}\left(y^{\prime}\right)\right],
\end{aligned}
$$

where we introduced the notation

$$
f_{1}(x)=\frac{1}{\left(x^{2}+\rho^{\prime 2}\right)}, \quad f_{2}(x)=\frac{\left(x_{\mu} x_{\nu}, i x^{2}\right)}{x^{2}\left(x^{2}+\rho^{\prime 2}\right)}, \quad g(x-y)=\frac{1}{4 \pi^{2}(x-y)^{2}},
$$

and assumed $\left(x_{\mu} x_{\nu}, i x^{2}\right)\left(y_{\mu} y_{\nu}, i y^{2}\right)=(x y)^{2}-x^{2} y^{2}$. In what follows we will use the notation $M_{s}^{2}(\vec{q}, m)$ for the dynamical color-octet scalar mass corresponding to the Matsubara mode $m$ with frequency $\omega_{m}=2 \pi m T$ in the three-momentum $\vec{q}$ representation. We are especially interested in the $m=0$ Matsubara mode, $M_{s}^{2}(\vec{q}, m=0) \equiv M_{s}^{2}(\vec{q}, T)$.

If we define the Fourier transformation to coordinate space as

$$
f_{1}(x-z)=\sum_{m=-\infty}^{\infty} \int \frac{d^{3} p}{(2 \pi)^{3}} \exp [i \vec{p}(\vec{x}-\vec{z})] \exp \left(2 \pi m(x-z)_{4} / \beta\right) f_{1}(\vec{p}, m),
$$

then the contribution of the first term in Eq. (35) can be rewritten as

$$
M_{s, 0,1}^{2}(q, T) \sim q^{4} f_{1}(\vec{q}, m=0) f_{1}(-\vec{q}, m=0)=q^{4} f_{1}^{2}(\vec{q}, 0),
$$

where

$$
\begin{aligned}
q^{2} f_{1}(q, 0) & \left.=q^{2} \rho^{\prime 2} \int_{-\beta / 2 \rho^{\prime}}^{\beta / 2 \rho^{\prime}} d x_{4} \int_{-\infty}^{\infty} d x_{2} d x_{3} \pi \exp \left(-q \rho^{\prime}\left(\sum_{i=2}^{4} x_{i}^{2}+1\right)^{1 / 2}\right)\right) \frac{1}{\left(\sum_{i=2}^{4} x_{i}^{2}+1\right)^{1 / 2}} \\
& \leq q^{2} \rho^{\prime 2} \int_{-\infty}^{\infty} d x_{4} d x_{2} d x_{3} \frac{\left.\pi \exp \left(-q \rho^{\prime}\left(\sum_{i=2}^{4} x_{i}^{2}+1\right)^{1 / 2}\right)\right)}{\left(\sum_{i=2}^{4} x_{i}^{2}+1\right)^{1 / 2}}=4 \pi^{2} q \rho^{\prime} K_{1}\left(q \rho^{\prime}\right),
\end{aligned}
$$

and $K_{1}(z)$ is a modified Bessel function of the second kind, with $\lim _{z \rightarrow 0} z K_{1}(z)=1$. Since temperature mildly affects the dynamical mass form factor, we can neglect this modification at small temperatures $T \leq T_{\mathrm{c}}$. Careful analysis shows that the second term in Eq. (35) and all of the other terms including $\Delta_{1}^{a b}$ and $\Delta_{2}^{a b}$ give zero or negligible contribution, so we finally obtain

$M_{s}(q, T) \approx M_{s, 0,1}(q, T)=\left[\frac{3 \bar{\rho}^{\prime 2}(T) n(T)}{\left(N_{\mathrm{c}}^{2}-1\right)} 4 \pi^{2}\right]^{1 / 2} F(q, T)$,

where

$$
F(0,0)=1, \quad F(q, T) \leq F(q, 0)=q \bar{\rho} K_{1}(q \bar{\rho}) .
$$

\section{GLUON PROPAGATOR AT NONZERO TEMPERATURE}

In this section we extend the calculations of the averaged full gluon propagator $\bar{S}_{\mu \nu}$, considered in Ref. [16], to the nonzero-temperature case. This is a rather straightforward task, since there all principal equations and their solutions were found in operator form. First, the solution of the Pobylitsa equation in operator form is

$$
\bar{S}_{\rho \nu}-S_{\rho \nu}^{0}=N\left(\bar{S}_{\rho \nu}^{I}-S_{\rho \nu}^{0}\right)+\mathcal{O}\left(n^{2}\right),
$$

where the free and single instanton gluon propagators [13] are given by

$$
\begin{aligned}
S_{\mu \nu}^{0} & =\left(\delta_{\mu \nu}-(1-\xi) p_{\mu} p_{\nu} / p^{2}\right) / p^{2}, \\
S_{\mu \nu}^{0-1} & =\delta_{\mu \nu} p^{2}-(1-1 / \xi) p_{\mu} p_{\nu},
\end{aligned}
$$




$$
S_{\mu \nu}^{I}=q_{\mu \nu \rho \sigma} P_{\rho}^{I} \Delta_{I}^{2} P_{\sigma}^{I}-(1-\xi) P_{\mu}^{I} \Delta_{I}^{2} P_{\nu}^{I},
$$

and we introduced the notation $q_{\mu \nu \rho \sigma}=\delta_{\mu \nu} \delta_{\rho \sigma}+\delta_{\mu \rho} \delta_{\nu \sigma}-$ $\delta_{\mu \sigma} \delta_{\nu \rho}+\epsilon_{\mu \nu \rho \sigma}$ (for the anti-instanton, case $+\epsilon_{\mu \nu \rho \sigma} \Rightarrow-\epsilon_{\mu \nu \rho \sigma}$ ). Equation (40) can be rewritten [cf. Eq. (21)] as

$$
\Pi_{\rho \nu} \equiv \bar{S}_{\rho \nu}^{-1}-S_{0, \rho \nu}^{-1}=N S_{\rho \sigma}^{0-1}\left(\bar{S}_{\sigma \mu}^{I}-S_{\sigma \mu}^{0}\right) S_{\mu \alpha}^{0-1}+\mathcal{O}\left(n^{2}\right) .
$$

At nonzero temperature, the most essential point is the lack of relativistic covariance, since Euclidian time is restricted to the interval $0 \leq x_{4} \leq \beta=1 / T$, and all of the bosonic fields (the background $A_{\mu}$, fluctuations $a_{\mu}$, and zero modes $\phi_{\mu}$ ) must be time-periodic functions with period $\beta, A_{\mu}\left(\vec{x}, x_{4}+\beta\right)=$ $A_{\mu}\left(\vec{x}, x_{4}\right)$. The operator form of the main equation (43) significantly simplifies our problem, since in the end we only have to calculate the matrix element of the operators between the time state $\left|t^{\prime}\right\rangle$ and periodic state $\left|t_{\beta}\right\rangle$ defined in Eq (19). Then, by means of Fourier transformations we can

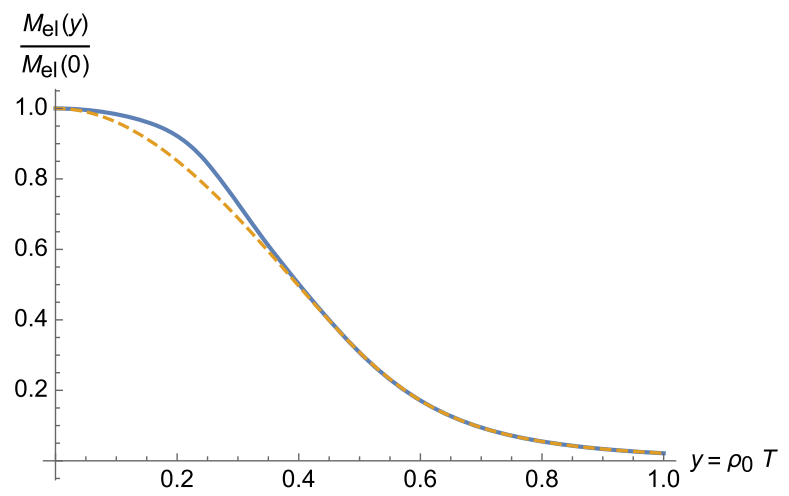

obtain the momentum representation of the propagators written in terms of three-momenta $\vec{k}$ and Matsubara modes $m\left[k_{4}=2 \pi m T, k_{\mu} \equiv\left(\vec{k}, k_{4}\right), k^{2}=\vec{k}^{2}+k_{4}^{2}\right]$.

We expect that the dominant contribution to $\Pi_{\nu \mu}$ will come from the large-distance asymptotics of the matrix elements of $S_{\nu \mu}^{I}-S^{0}{ }_{\nu \mu}$. In coordinate space, by comparing the effects from $i \partial_{\mu}$ and the multiplication by $A_{\mu}^{I}$ in Eq. (42), we conclude that the dominant asymptotic contribution to $S_{\nu \mu}^{I}-S^{0}{ }_{\nu \mu}$ in Eq. (43) comes from the term

$$
p_{\rho}\left(\left(\Delta_{I}-\Delta_{0}\right) \Delta_{0}+\Delta_{0}\left(\Delta_{I}-\Delta_{0}\right)\right) p_{\sigma} .
$$

in particular, from $p_{\rho}$ [the most slowly decreasing part of Eq. (44)]. The term (44) will also give the dominant contribution to $\Pi_{\nu \mu}$. So, Eq. (43) reduces to

$$
\Pi_{\mu \nu}=2 N p^{2}\left(\Delta_{I, 0}-\Delta_{0}\right)\left(p^{2} \delta_{\mu \nu}-(1-1 / \xi) p_{\mu} p_{\nu}\right) .
$$

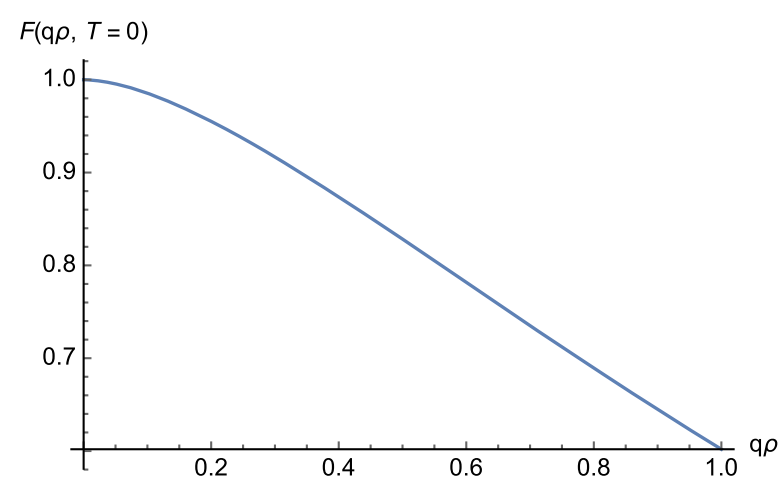

FIG. 2. Left: Temperature dependence of the "electric" gluon dynamical mass $M_{\mathrm{el}}(0, T) / M_{\mathrm{el}}(0,0)$. The solid line was evaluated using the modification $A_{N_{\mathrm{c}}} \rightarrow A_{N_{\mathrm{c}}} \Theta_{\Delta y}\left(y-y_{\mathrm{c}}\right)$ [see Eqs. (9) and (14)] and interpolates smoothly between no suppression below the critical temperature $T_{\mathrm{c}}=150 \mathrm{MeV}$ and full suppression above it, with the width of the interpolating region $\Delta T=0.3 T_{\mathrm{c}}$ [5]. At small $T \leq T_{\mathrm{c}}$ the solid line corresponds to $M_{\mathrm{el}}(0, T) / M_{\mathrm{el}}(0,0)=\bar{\rho}^{\prime}(T) / \bar{\rho}(T)=\left(1-1 / 6 \pi^{2} \bar{\rho}_{0}{ }^{2} T^{2}\right)$. The dashed line corresponds to full suppression over the whole region of $T\left[A_{N_{\mathrm{c}}}=\right.$ const in Eq. (9)]. In both plots we use the phenomenological estimates $M_{\mathrm{el}}(0,0)=362 \mathrm{MeV}$, $\bar{\rho}(0)=1 / 3$, fm and $n(0)=1 \mathrm{fm}^{-4}$. Right: Form factor of the dynamical mass $F(q, 0)$ [Eq. (39)].
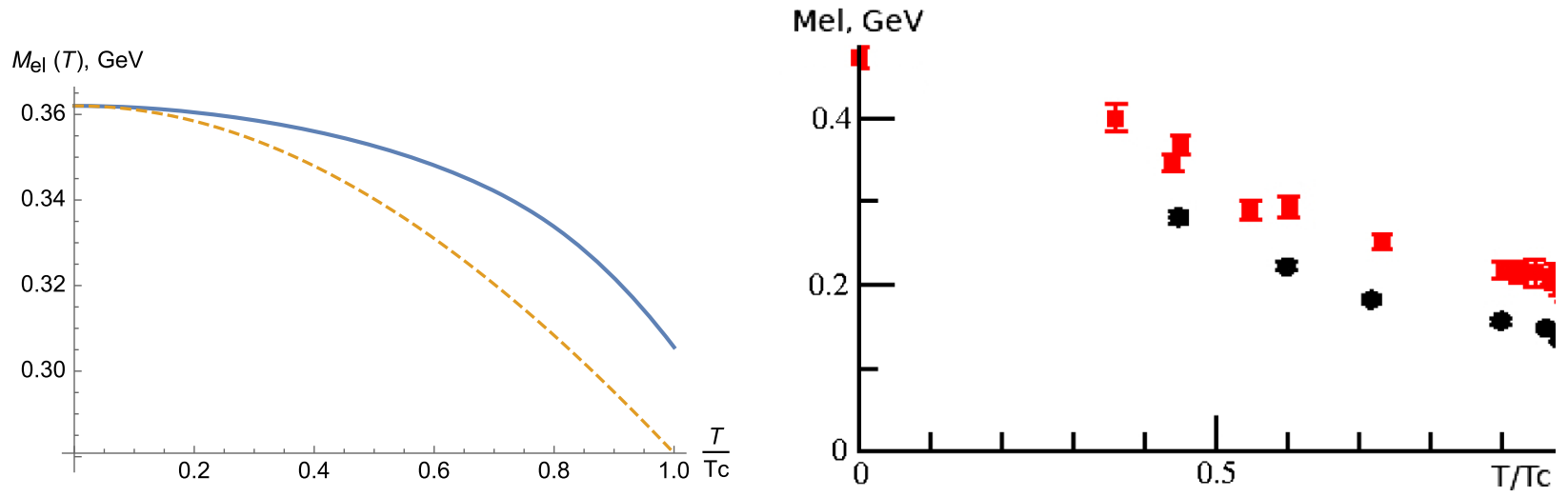

FIG. 3. Comparison of the "electric" gluon dynamical masses from the ILM and lattice measurements. Left: $T$ dependences of $M_{\mathrm{el}}(0, T)$ in the region $0<T<T_{\mathrm{c}}$ within the ILM (all definitions are the same as in Fig. 2). Right: Lattice measurement results for the same quantity taken at the scale $2 \mathrm{GeV}$ [9-12]. (The right plot is a part of Fig. 12 from Ref. [11]; see the caption of that figure for further details.) 
By definition, the square of the "electric" gluon mass $M_{\mathrm{el}}^{2}(|\vec{k}|, T)$ is related to $\Pi_{\mu \nu}$ as $M_{\mathrm{el}}^{2}(|\vec{k}|, T)=\Pi_{44}\left(\vec{k}, k_{4}=0\right)$. Comparing it with Eq. (34), we conclude that $M_{\mathrm{el}}^{2}(|\vec{k}|, T)=$ $2 M_{s}^{2}(|\vec{k}|, T)$ is gauge invariant ( $\xi$ independent), and its $T$ and $q$ dependences are represented in Fig. 2. It is obvious that $M_{\mathrm{el}}^{2}(|\vec{k}|, T=0)=M_{g}(|\vec{k}|)$, where the gauge-invariant dynamical gluon mass $M_{g}$ was obtained in Ref. [16]. Using the phenomenological values of $\bar{\rho}$ and $n$ at $T=0$, we obtain $M_{\mathrm{el}}(0,0)=362 \mathrm{MeV}$.

From Fig. 3 we see that the most natural explanation for a nonzero "electric" gluon dynamical mass in the region $T<T_{\mathrm{c}}$ seen in lattice measurements $[9,12]$ is given by the ILM, since the ILM is able to (at least qualitatively) reproduce its value at $T=0$ and its $T$ dependences.

\section{SUMMARY AND DISCUSSION}

In this paper we extended the calculations of the dynamical gluon mass in the ILM [16] to nonzero temperature and studied the so-called "electric" gluon mass $M_{\mathrm{el}}(q, T)$, which corresponds to the $\Pi_{44}$ component of the polarization operator. We also analyzed the temperature ( $T$ ) dependence of the main parameters of the ILM: the average instanton size $\bar{\rho}(T)$ and instanton density $n(T)$. We found that they are homogeneously decreasing functions of temperature due to the influence of thermal gluon fluctuations [6]. Our findings agree with lattice investigations [19], which demonstrated that $\bar{\rho}(T)$ and $n(T)$ decrease rapidly for $T \geq T_{\mathrm{c}}$, where $T_{\mathrm{c}}$ is the critical temperature. For temperatures below the critical temperature $T_{\mathrm{c}}$, these functions are almost constant, and we took this scenario into account by neglecting the contributions of thermal gluon fluctuations at low temperatures $T \leq T_{\mathrm{c}}$ [5]. These scenarios are compared in Fig. 1.

In order to find the gluon propagator in the ILM background field at nonzero temperature $T \neq 0$, we solved the gluon zero-mode problem and averaged the full gluon propagator over the collective coordinates of all instantons. This was done in the framework developed in our previous paper [16] and extended to the nonzero-temperature case. First, we evaluated the "electric" color-octet scalar dynamical mass $M_{s}(q, T)$ as a function of the threemomentum $\vec{q}$ and temperature $T$. The solution of the zero-mode problem yields $M_{\mathrm{el}}^{2}(q, T)=2 M_{s}^{2}(q, T)$, which allowed us to relate. The final results for the "electric" gluon dynamical mass $M_{\mathrm{el}}(q, T)$ are presented in Fig. 2.

It is interesting to compare our result for the dynamical "electric" gluon mass $M_{\mathrm{el}}$ with the result from lattice calculations (see Fig. 3), which observed that $M_{\mathrm{el}}(0, T)$ is a decreasing function of $T$ for $T \leq T_{\mathrm{c}}$ (in agreement with the ILM) and an increasing function of $T$ above the confinement-deconfinement phase transition $[9,12]$. It is clear from Eq. (45) that the "electric" gluon mass is a gauge-independent ( $\xi$-independent) quantity. Also, the natural scale for the ILM is $\rho^{-1} \sim 1 \mathrm{GeV}$. On the other hand, the lattice measurements of the "electric" gluon mass shown in Fig. 12 of Ref. [11] were given in Landau gauge $(\xi=0)$ and at the scale $2 \mathrm{GeV}$. So, due to the scale difference the comparison presented in Fig. 3 is only qualitative. Here it is most important that lattice measurements demonstrated a nonzero "electric" gluon mass at low temperatures. The growth of $M_{\mathrm{el}}(0, T)$ for $T \geq T_{\mathrm{c}}$ may be explained by the perturbative thermal gluon correction and it is expected to have an almost linear functional dependence, $M_{\text {pert,el }}(0, T) \sim T$. Since thermal gluons are incorporated into our framework, it is probably easy to reproduce the lattice measurements of the dynamical "electric" gluon mass within the ILM model over the whole temperature region.

In the future, we hope to apply our results to the calculation of the temperature dependences of the heavy quarkonium properties, and to extend the present calculation to the dynamical "magnetic" gluon mass case.

\section{ACKNOWLEDGMENTS}

M. M. is thankful to Marat Siddikov and Boris Kopeliovich for the useful and helpful communications. This work is partially supported by Fondecyt (Chile) Grant No. 1140377 and Grant No. OT-F-2-010 of the Uzbekistan Ministry for Innovational Development.
[1] A. A. Belavin, A. M. Polyakov, A. S. Schwartz, and Y. S. Tyupkin, Phys. Lett. 59B, 85 (1975).

[2] B. J. Harrington and H. K. Shepard, Phys. Rev. D 17, 2122 (1978).

[3] D. I. Diakonov and V. Yu. Petrov, Nucl. Phys. B245, 259 (1984).

[4] D. Diakonov, Prog. Part. Nucl. Phys. 51, 173 (2003).

[5] T. Schaefer and E. Shuryak, Rev. Mod. Phys. 70, 323 (1998).
[6] D. I. Diakonov and A. D. Mirlin, Phys. Lett. B 203, 299 (1988).

[7] R. Larsen and E. Shuryak, Phys. Rev. D 96, 034508 (2017).

[8] Y. Liu, E. Shuryak, and I. Zahed, Phys. Rev. D 98, 014023 (2018).

[9] P. J. Silva, O. Oliveira, D. Dudal, P. Bicudo, and N. Cardoso, Few Body Syst. 58, 127 (2017). 
[10] P. Bicudo, O. Oliveira, P. J. Silva, and N. Cardoso, Proc. Sci., LATTICE2013 (2014) 368.

[11] P. J. Silva, O. Oliveira, P. Bicudo, and N. Cardoso, Phys. Rev. D 89, 074503 (2014).

[12] A. Maas, J. M. Pawlowski, L. von Smekal, and D. Spielmann, Phys. Rev. D 85, 034037 (2012).

[13] L. S. Brown, R. D. Carlitz, D. B. Creamer, and C. Lee, Phys. Rev. D 17, 1583 (1978).

[14] L. S. Brown and C. Lee, Phys. Rev. D 18, 2180 (1978).

[15] P. V. Pobylitsa, Phys. Lett. B 226, 387 (1989).
[16] M. Musakhanov and O. Egamberdiev, Phys. Lett. B 779, 206 (2018).

[17] S. i. Nam, Phys. Rev. D 87, 116003 (2013).

[18] D. J. Gross, R. D. Pisarski, and L. G. Yaffe, Rev. Mod. Phys. 53, 43 (1981).

[19] M. C. Chu and S. Schramm, Phys. Rev. D 51, 4580 (1995); M.-C. Chu, S. M. Ouellette, S. Schramm, and R. Seki, Phys. Rev. D 62, 094508 (2000).

[20] J. Schwinger, Phys. Rev. 82, 664 (1951). 